\title{
NEURO-FUZZY SYSTEM TO CONTROL THE FAST FERRY VERTICAL ACCELERATION
}

\author{
R. López, M. Santos \\ Dept. Arquitectura de Computadores y Automática \\ Facultad de CC. Físicas, Universidad Complutense de Madrid \\ 28040-Madrid. Spain \\ msantos@dacya.ucm.es
}

\begin{abstract}
This paper shows the design of a neuro-fuzzy system to control the vertical motion of a TF-120 fast ferry, so that to improve the passengers comfort by reducing the main cause of seasickness. The aim of this controller is to decrease the pitch acceleration by controlling the position of some actuators, varying their working angles. The controller is based on a fuzzy model of the ship behaviour. It has been simulation tested in regular and irregular waves with satisfactory results. The MSI (Motion Sickness Incidence), has been evaluated to prove the efficiency of this strategy. Although neuro-fuzzy controllers have been widely used before, the application of this strategy to control marine systems is novel. Copyright (C) 2002 IFAC
\end{abstract}

Keywords: Neuro-Fuzzy Control, Ship Control, Marine Systems, Vertical Motion, Hydraulic Actuators.

\section{INTRODUCTION}

The aim of this work is to design a fuzzy controller in order to reduce the vertical motion of a high-speed craft. A crucial goal dealing with fast ferries is to stabilise the motion of the ship for some purposes, such as the improvement of the comfort of the passengers and the crew, while maintaining the speed. The final target is to reduce the MSI (Motion Sickness Incidence), an index used to measure the seasickness, which is mainly caused by the impact of the vertical oscillations.

An accurate mathematical model is difficult to achieve because of the strongly non-linear nature of these kind of marine systems. In addition, the uncertainty that comes from the sea waves encourages to deal with wide broad controllers, which can incorporate expert knowledge. Because of that, a neuro-fuzzy controller has been designed to include the available experimental knowledge about the behaviour of the system that is wanted to be controlled.

One of the principal knowledge sources for modelling and control is the collected experimental data of the performance of a high-speed craft in regular waves. These data has been obtained by carrying out some experiments with a small replica of the ship, and has been then computed by a numeric program called PRECAL, all of this provided by a specialised towing tank (Cehipar, 1998). The physical studies of the vertical dynamics of the craft and the model of the actuators (flaps and T-foils) have also contributed with useful information to the development of the fuzzy system.

A neuro-fuzzy model of the ship has been already developed (López and Santos, 2002), based on this experimental knowledge and applying adaptive neuro-fuzzy inference. Taking this model as a starting point, the control strategy is defined.

The fuzzy controller is focused in reducing the vertical motion. The pitch acceleration is considered and the neuro-fuzzy system controls the position of the control surfaces that have been added to the system for decreasing pitch motion. In this case, the fins are two flaps at stern and a T-foil at bow.

The controller has been tested in a simulation environment, in regular and irregular waves, with 
encouraging results. The implemented correction is calculated in order to compare it with other control strategies and to compute the improvement by the MSI index.

The paper is structured as follows. Section 2 summarises the principal aspects of the available fuzzy model of the ship behaviour. Section 3 presents the actuators to be controlled. In Section 4, the neuro-fuzzy system is designed for controlling both, the amplitude and the phase of the actuators, and some simulation results are presented. In Section 5, using the MSI index, an evaluation of the controller has been performed.

\section{NEURO-FUZZY MODEL}

The research deals with a fast ferry called "Silvia Ana", an aluminium-made craft with a deep V hull, $126 \mathrm{~m}$ long, $14.696 \mathrm{~m}$ beam, 2.405 draught, 475 tons dead weight, and 1250 passengers capacity (Anonymous, 1996; Anonymous, 1998). A scale down dimension replica is available to make some experiments.

The main source of knowledge is always a deep knowledge of the physics of the problem, both the marine systems and the waves (Lewis, 1989; Lloyd, 1998; Fossen, 1994).

On the other hand, using a small replica of the fast ferry we are working with, a series of experiments have been performed by a towing tank institution, CEHIPAR (Cehipar, 2000). The obtained experimental results and the previous information allowed us to develop a neuro-fuzzy model (López and Santos, 2002).

In addition, CEHIPAR can supply simulated data of the ship performance by using the computer program PRECAL (based on finite elements) to validate the model and to test the controller (Cehipar, 1998).

The model focuses on heave and pitch motions, analysing the vertical accelerations of the marine system. It has three inputs:

- the sea state number (SSN), according to the Word Meteorological Organisation (WMO) -given by the modal frequency, $\omega_{0}$, or the wavelength-. The modal frequency of the waves is calculated by means of the observed wave height, $\mathrm{H}$, applying the PiersonMoskowitz spectrum.

- the ship speed, U,

- the heading angle, $\mu$, angle relative to the direction of propagation of a train of regular waves.

The outputs are the heave and pitch amplitude and phase, and the total pitch moment.
The fuzzy non-linear model shows good pattern with experimental and simulated data in regular waves. Its modular structure allows to connect different types of controllers.

\section{ACTUATORS}

The main goal of the actuators is to reduce the vertical motion of the ship by using the lift forces that are caused by varying their opening angles. The expression of this force is:

$$
\mathrm{L}=\rho \mathrm{SU}^{2} \frac{\mathrm{dC}_{\mathrm{L}}}{\mathrm{d} \alpha} \alpha
$$

where $C_{L}$ is the lift coefficient, $\rho$ is the fluid density $\left(1.025 \mathrm{MTm} / \mathrm{m}^{3}\right), \mathrm{S}$ is the area of the control surface, $\mathrm{U}$ the ship speed, and $\alpha$ is the working angle of the actuator. For a fixed ship speed, U, the lift force only depends on the working angle, $\alpha$.

It is essential to know how these fins operate to design the control rules. The characteristics and position of the two added flaps at stern and the T-foil at bow are shown in Table 1, according to the dimension of the particular ship we are working with.

Table 1: Physical characteristics of the actuators

\begin{tabular}{lcc} 
& flap & T-foil \\
\hline area $\left(\mathrm{m}^{2}\right)$ & 11 & 13,5 \\
maximum angle $\left({ }^{\circ}\right)$ & 15 & $+15 /-15$ \\
lift coefficient $\left(\mathrm{kN} /{ }^{\circ} / \mathrm{m}^{2} / \mathrm{knot}^{2}\right)$ & $9,19 \mathrm{E}-03$ & $6,09 \mathrm{E}-03$ \\
rotational max.speed $(\% / \mathrm{s})$ & 13,5 & 13,5 \\
distance to the $\operatorname{cog}(\mathrm{m})$ & 41,6 & 58,4 \\
\hline
\end{tabular}

Both actuators rotate around its bolt. The motion of the flaps is limited upwards. The wings of the T-foil can freely move upwards and downwards. As it is possible to notice, these control surfaces work underwater.

In order to estimate the maximum pitch correction that these fins can provide, the maximum angle that they can achieve must be calculated.

Assuming that $\omega_{\mathrm{f}}$ and $\omega_{\mathrm{T}}$ are the rate of oscillation of the flaps and the T-foil, respectively, and $13.5 \%$ is the rotational speed, the maximum opening angles will be,

$$
\varphi_{f M A X}=\min \left(15,13.5 \frac{T}{2}\right)=\min \left(15,13.5 \frac{\pi}{\left|\omega_{f}\right|}\right)
$$

for the flaps, and for the T-foil,

$$
\varphi_{T_{M A X}}=\min \left(15,13.5 \frac{T}{2}\right)=\min \left(15,13.5 \frac{\pi}{\left|\omega_{T}\right|}\right)
$$


Then, the amplitudes of the angle of incidence that they can reach can be expressed as a function of these values,

$$
\begin{gathered}
\varphi_{f}=\frac{1}{2} \varphi_{f M A X} \sin \left(\omega_{f} t\right)+\frac{1}{2} \varphi_{f M A X} \\
\varphi_{T}=\frac{1}{2} \varphi_{T M A X} \sin \left(\omega_{T} t\right)
\end{gathered}
$$

Now, if they are wanted to be effective, they should oscillate at the same rate that the encounter frequency, $\omega_{\mathrm{e}}$. The encounter frequency is the main oscillation rate of the system, and it is defined as the frequency at which the ship and a train of regular waves meet. It is a function of the frequency of the waves, $\omega_{0}$, the speed of the craft, $U$, and the heading angle, $\mu$.

Therefore, the real maximum angle that the actuators can reach has been calculated as a function of the encounter frequency. When plotting $\varphi_{\mathrm{fMAX}}$ and $\varphi_{\text {TMAX }}$ vs. the encounter frequency, $\omega_{\mathrm{e}}$, it is posible to prove that, for different values of $\omega_{\mathrm{e}}$, the flap reachs $15^{\circ}$. But the $\mathrm{T}$-foil only varies between $\pm 15^{\circ}$ for frequencies lower than $1.5 \mathrm{rad} / \mathrm{s}$. For higher frequencies, the total opening angle of the T-foil decreases. That means that the $\mathrm{T}$-foil does not reach $30^{\circ}$ in practice in all the range of frequencies we are interested in: 0-2 rad/s. That result will be considered in the implementation of the controller.

To compute the total pitch moment correction provided by the actuators, the lift forces are going to be expressed as a function of the parameters of the ship motion.

The speed $U$ can be expressed in terms of its components as,

$$
\begin{gathered}
\vec{U}_{f}=\vec{U}_{f X}+\vec{U}_{f Y}=\vec{U}_{\text {ship }} \\
\vec{U}_{T}=\vec{U}_{T X}+\vec{U}_{T Y}=\vec{U}_{\text {ship }}+\vec{U}_{\text {pitch_T-foil }}+\vec{U}_{\text {heave }}
\end{gathered}
$$

where the subindex $f$ means flap contribution and $T$ refers to T-foil contribution.

Moreover

$$
\begin{gathered}
\vec{U}_{\text {ship }}=U_{\text {ship }} \vec{i} \\
\vec{U}_{\text {pitch_T-foil }}=R_{T} \dot{x}_{5} \sin \left(x_{5}\right) \vec{i}+R_{T} \dot{x}_{5} \cos \left(x_{5}\right) \vec{j} \\
\vec{U}_{\text {heave }}=\dot{x}_{3} \vec{j}
\end{gathered}
$$

where $\mathrm{R}_{\mathrm{T}}$ is the radio of $\mathrm{T}$-foil movement.

Taking into account Figure 1 and Figure 2, which show the actuators motion, it is possible to establish some equations to define the movement of their angles.
Supposing that the fluid flow coming to the flap and the longitudinal axis run parallel, the working angles, expressed as a function of the opening angles, $\varphi$, and the pitch, $x_{5}$, are,

$$
\begin{gathered}
\alpha_{f}=\varphi_{f}+x_{5} \\
\alpha_{T}=\theta_{T}+\varphi_{T}-x_{5}
\end{gathered}
$$

Considering that the angle between the lift force, L, and the normal line to the longitudinal axis of the ship is $x_{5}-\theta_{\mathrm{T}}$ (see Figure 1),

$$
\theta_{T}=\arctan \frac{R_{T} \dot{x}_{5} \cos \left(x_{5}\right)+\dot{x}_{3}}{\mathrm{U}-R_{T} \dot{x}_{5} \sin \left(x_{5}\right)}
$$

that, for small values of $x_{5}$, it is possible to approximate to

$$
\theta_{T} \approx \arctan \frac{\left(R_{T} x_{5 M A X}+x_{3 M A X}\right) \omega_{e} \cos \left(\omega_{e} t\right)}{\mathrm{U}-\frac{R_{T}}{2} x_{5 M A X}^{2} \omega_{e} \sin \left(2 \omega_{e} t\right)}
$$

The component $\theta$ appears only at bow, where the Tfoil is placed, because of the vertical component of the fluid flowing.

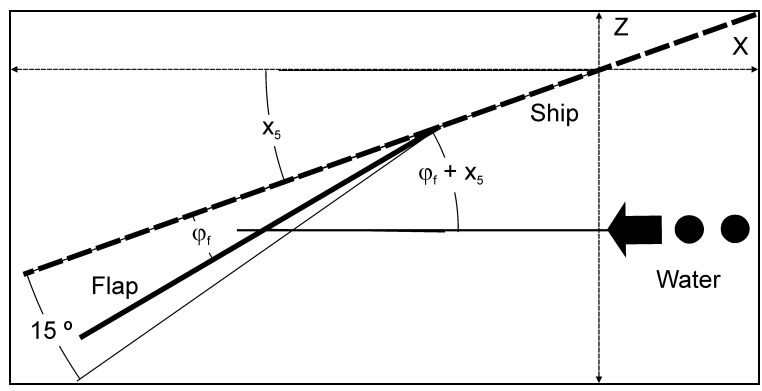

Fig. 1: Flap motion

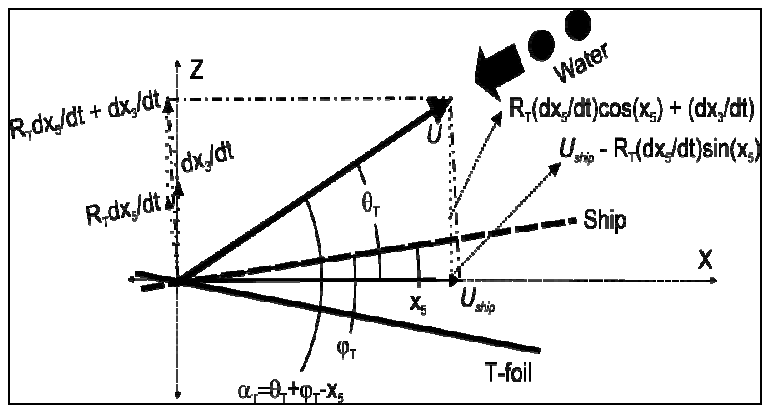

Fig. 2: T-foil motion

Applying equation (1) and substituting the angles, the lift forces that can be applied will be then,

$$
\begin{gathered}
\mathrm{L}_{f}=k_{f}\left|\vec{U}_{f}\right|^{2} \alpha_{f} \\
\mathrm{~L}_{T}=k_{T}\left|\overrightarrow{\mathrm{U}}_{T}\right|^{2} \alpha_{T} \cos \left(x_{5}-\theta_{T}\right) \approx k_{T}\left|\overrightarrow{\mathrm{U}}_{T}\right|^{2} \alpha_{T}
\end{gathered}
$$

where 


$$
\begin{aligned}
& k_{f}=\rho S_{f} \frac{d C_{L}}{d \alpha_{f}}=0.104 \\
& k_{T}=\rho \mathrm{S}_{T} \frac{d \mathrm{C}_{\mathrm{L}}}{d \alpha_{T}}=0.100
\end{aligned}
$$

Therefore, the maximum pitch correction is given by the moment provided by the flaps and the T-foil, i.e.,

$$
\mathrm{CMP}=\mathrm{M}_{\mathrm{f}}+\mathrm{M}_{\mathrm{T}}=\mathrm{R}_{\mathrm{f}} \mathrm{L}_{\mathrm{f}}+\mathrm{R}_{\mathrm{T}} \mathrm{L}_{\mathrm{T}}
$$

where $R_{f}$ and $R_{T}$ are the radio of the flap and T-foil movement, as are defined in Table 1 , and $\mathrm{L}_{\mathrm{f}}$ and $\mathrm{L}_{\mathrm{T}}$ are calculated by (4) and (5).

Table 2 summarises, for different sea states, the mean value of the maximum pitch correction (CMP) that is possible to achieve -by applying (6)-, and the maximum pitch moment (MMP), calculated by the model (without actuators).

Table 2: Mean value of the maximum pitch correction

\begin{tabular}{cccc} 
SNN & CMP & MMP & $\boldsymbol{\Delta} \%$ \\
\hline 3 & 93.931 & 375,431 & 25 \\
4 & 93,180 & 445,592 & 21 \\
5 & 101,741 & 401,129 & 25 \\
6 & 97,590 & 202,899 & 48 \\
7 & 94,493 & 131,496 & 72 \\
\hline
\end{tabular}

Figure 3 show the total pitch moment (solid line) and the correction (dashed line) vs. the encounter frequency, for a particular situation (SNN of 4). The same experiments have been also realised for different SNN (3 to 7) to show the total pitch moment and the correction vs. the ship speed in the advance direction.

The improvement is remarkably in all the cases, specially for high sea states.

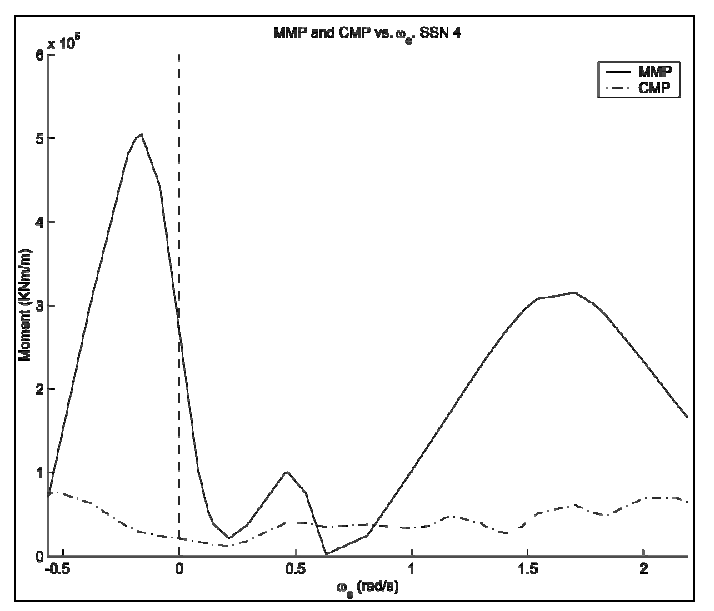

Fig. 3: Total pitch moment and correction vs. the encounter frequency, $\omega_{\mathrm{e}}$
It is possible to notice that for some frequencies, the correction can be larger than the total moment. This must be taken into account to design the control rules.

\section{NEURO-FUZZY CONTROL}

The output of the controller is the position of the flap and the T-foil, in order to reduce the pitch moment. The inputs of the controller are the sea state (given by the modal wave frequency), the ship speed, U, and the heading angle, $\mu$. Another input is needed to set the phase. The pitch phase, $\varphi_{5}$, is provided by the fuzzy model of the ship, which is demanded by the controller.

The implemented FIS (Fuzzy Inference Systems) calculates the working angles of the flaps and the Tfoil, bounding them to the physical constraints of $15^{\circ}$ and $30^{\circ}$ (see Table 1 ). The controller computes the output signals as,

$$
\begin{gathered}
S_{f}=\frac{1}{2} \varphi_{f} \sin \left(\varphi_{5}\right)+\frac{1}{2} \varphi_{f} \\
S_{T}=\frac{1}{2} \varphi_{T} \sin \left(\varphi_{5}\right)
\end{gathered}
$$

That is, the fuzzy controller gives the position (amplitude of the angle) of the flap and T-foil.

The controller has been tested by simulation. The structure of the simulation system consist of a series of modular blocks. The first block refers to the waves, that are characterised by the modal frequency, which is calculated from the observed wave height by applying the Pierson-Moskowitz formula. The input is then the wave height, and the output is the corresponding modal frequency that identifies the Sea State number.

The next block is called Ship, and it represents the fuzzy model of the ship. Its three inputs are: the output of the previous block -the modal frequency of the waves-, the ship speed and the heading angle.

One of its output, the pitch phase, is required as an input to the next block, the controller. The other inputs of the controler are the parameters of the motion (ship speed, waves direction and modal wave frequency). The outputs of the control system, i.e., the position of the T-foil and the position of the flap, are introduced in the model of the actuators.

\subsection{Simulation Results}

First of all, the controller has been proved in regular waves, for different SSN (3 to 7), at 20, 30 and 40 knots, and different heading $\left(0^{\circ}, 60^{\circ}, 120^{\circ}\right.$ and $\left.180^{\circ}\right)$. Figure 4 shows an example for waves of $3.78 \mathrm{~m}$ 
height (SSN 5), ship speed $U=40$ knots, and heading angle $\mu=180^{\circ}$.

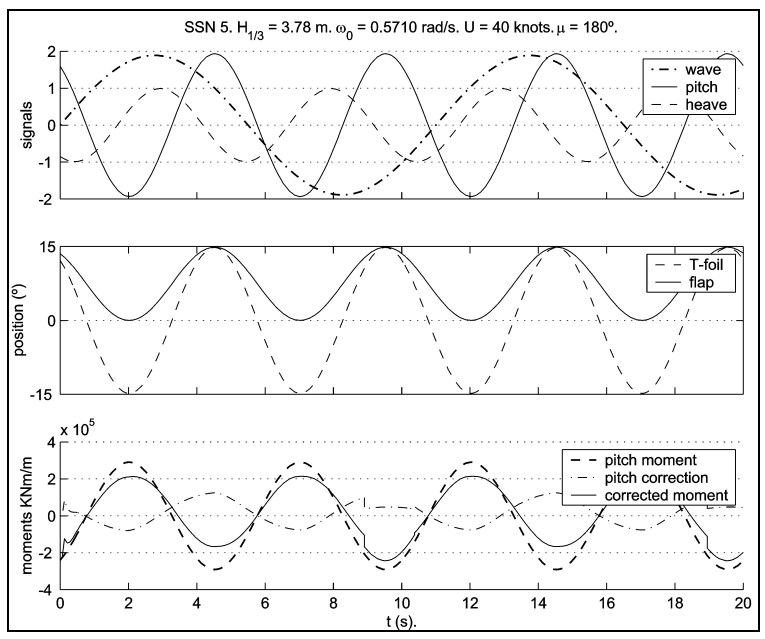

Fig. 4: Simulation results

The ratio between the moment correction and the total moment is presented in Table 3. Except for some of the values that exceed the 100\% (shaded), the results are quite similar to the expected correction (Table 2).

Table 3: Ratio (\%) between the moment correction and the total moment

\begin{tabular}{|c|c|c|c|c|c|c|c|c|c|c|c|c|c|}
\hline \multirow{2}{*}{$\frac{\text { SNN }}{\left(^{\circ}\right)}$} & \multicolumn{4}{|c|}{20 knots } & \multicolumn{4}{|c|}{30 knots } & \multicolumn{4}{|c|}{40 knots } & \multirow[t]{2}{*}{$\Delta$} \\
\hline & 0 & 60 & 120 & 180 & 0 & 60 & 120 & 180 & 0 & 60 & 120 & 180 & \\
\hline 3 & & 74 & 11 & 36 & & 30 & 25 & 12 & & 10 & 38 & 5 & 27 \\
\hline 4 & 72 & 41 & 13 & 11 & 33 & 81 & 19 & 22 & & 30 & 23 & 45 & 35 \\
\hline 5 & 56 & 50 & 30 & 15 & 62 & 87 & 43 & 19 & 9 & & 51 & 27 & 41 \\
\hline 6 & 61 & 68 & 31 & 20 & 59 & & 63 & 30 & 13 & & 100 & 55 & 50 \\
\hline 7 & 76 & & 70 & 40 & 89 & & 96 & 55 & 70 & 99 & & 79 & 75 \\
\hline
\end{tabular}

Although both, the model and the controller, have been designed for ideal waves, some experiments have been carried out for irregular waves, varying the observed height (SSN of 4, 5 and 6), the ship speed (20, 30 and 40 knots for each one), and $180^{\circ}$ heading.

A phase correction has been added to take into account that the pitch acceleration is out of phase $180^{\circ}$.

The total moment is reduced by applying the moment correction provided by the actuators, although these actuators do not reach the maximum opening angle in some cases because of the change of phase.

Figure 5 shows an example of the simulation for irregular waves of $2.49 \mathrm{~m}$ height (corresponding to SSN of 5), ship speed $U=30$ knots, and heading angle $\mu=180^{\circ}$.

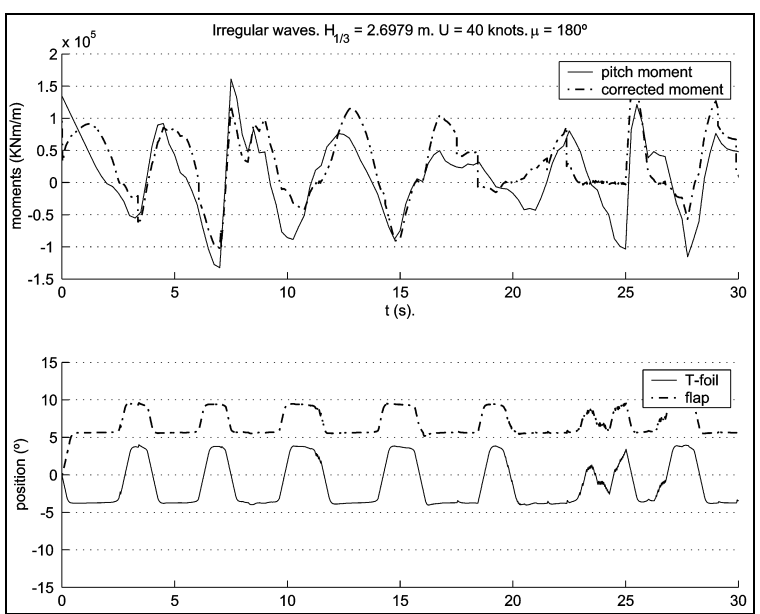

Fig. 5: Simulation results with irregular waves

To improve the results, the position of the centre of the oscillation has been changed. In fact, the actuators can oscillate around the mean position $\left(7.5^{\circ}\right.$ for the flap and $0^{\circ}$ for the T-foil) or around another position (trimming). Because of the actuators are not able to reach their maximum opening angles, changing the oscillation centre could help to compute the final pitch acceleration more accurately. For instance, $12.5^{\circ}$ for the flap and $-10^{\circ}$ for the T-foil as trimmed positions can be adequate values, as it has been proved experimentally.

\section{EVALUATION}

To evaluate the efficiency of the fuzzy model and the controller, a standard test is to measure the acceleration suffered by the passenger who is situated in the worst position in the ship, where the vertical acceleration is the largest. For this value, the MSI (motion sickness incidence) (O'Hanlon and McCawley, 1974) is computed by applying the expression given by Cruz (2000).

The simulation has been carried out considering as controller inputs both, the pitch and the heave signals (provided either by the fuzzy model or by real experiments). The results are quite similar, but the fuzzy controller performs slightly better when the inputs are the experimental data.

Table 4: MSI for the worst situated passenger

\begin{tabular}{|c|c|c|c|c|c|c|c|c|}
\hline \multirow[t]{2}{*}{ SNN } & \multirow[t]{2}{*}{$\mathrm{U}$} & \multirow[t]{2}{*}{$\mathrm{H}_{1 / 3}$} & \multirow[t]{2}{*}{$\omega o$} & \multirow[t]{2}{*}{$\omega e$} & \multirow{2}{*}{$\begin{array}{l}\text { without } \\
\text { actuator }\end{array}$} & \multirow{2}{*}{$\begin{array}{l}\text { without } \\
\text { controller }\end{array}$} & \multicolumn{2}{|c|}{ with controller } \\
\hline & & & & & & & $\begin{array}{c}\text { no } \\
\text { trimmer } \\
\end{array}$ & trimmer \\
\hline 4 & 20 & 1.67 & 0.86 & 1.64 & 0.11 & 0.10 & 0.10 & 0.09 \\
\hline 4 & 30 & 1.25 & 0.99 & 2.54 & 0.03 & 0.03 & 0.03 & $0.02-3$ \\
\hline 4 & 40 & 1.51 & 0.90 & 2.61 & 0.09 & 0.07 & 0.08 & 0.07 \\
\hline 5 & 20 & 2.74 & 0.67 & 1.14 & 0.32 & 0.31 & 0.31 & 0.30 \\
\hline 5 & 30 & 2.49 & 0.70 & 1.48 & 0.41 & 0.38 & 0.37 & 0.36 \\
\hline 5 & 40 & 2.70 & 0.68 & 1.63 & 0.53 & 0.49 & 0.48 & 0.46 \\
\hline 6 & 20 & 3.85 & 0.57 & 0.90 & 0.42 & 0.42 & 0.41 & 0.40 \\
\hline 6 & 30 & 3.51 & 0.59 & 1.14 & 0.55 & 0.53 & 0.52 & 0.51 \\
\hline 6 & 40 & 3.44 & 0.60 & 1.35 & 0.65 & 0.63 & 0.61 & 0.61 \\
\hline
\end{tabular}


As it has been said, the actuators do not achieve their maximum opening angles. Therefore, to set a trimmer for the oscillation centre, for a fixed encounter frequency, improves the control, as it is also shown in Table 4, which summarises the MSI, for the worst situated passenger with the bestobtained control.

To complete the study, basin test are being carried out although there are no results yet. We are employing an industrial PC to test the control algorithm with the scale down replica. A visual CASE tool for real time automatic control code generation, called EdROOM, is used, which runs under Windows and allows us to make easy the redesign of the control program. This software tool has been developed in our work group (Polo, et all., 2001).

The simulation results are very promising in terms of improving the comfort and avoiding the seasickness by decreasing the vertical acceleration.

As the normal sailing conditions (small or moderate waves) means low pitch acceleration, reducing the vertical motion by means of this control strategy enlarges the operational range of the ship, to carry passengers.

\section{CONCLUSIONS}

A neuro-fuzzy non-linear model has been previously obtained for a fast ferry. Based on physical principles, experimental and simulated data, and expert knowledge, the model has been developed for pitch motion and heading seas.

Actuators have been coupled to the model of the craft and neuro-fuzzy control strategies have been tested to reduce the vertical motion. By studying the operation of the control surfaces, the maximum pitch correction to the total pitch moment has been applied.

The performance of the controller has been tested in regular and irregular waves. Results are encouraging, especially because the vertical acceleration can be notably reduced by changing the centre of actuator oscillation.

Although neuro-fuzzy controllers have been widely used before, as far as we know, the application of this strategy to control marine systems is novel.

The main application of this control is the improvement of the passenger comfort by reducing the sea-sickness caused by the vertical motion. Therefore, the operational range of the ship, to carry passengers, is enlarged.

\section{ACKNOWLEDGEMENTS}

Authors would like to thank the support of the CICYT Spanish Committee (Projects TAP97-0607C03-01 and DPI 2000-0386-C03-01) and the collaboration of the CEHIPAR staff.

\section{REFERENCES}

Anonymous (1996). 126 m Long Spanish Fast Ferry Launched. Fast Ferries, Sept, 19-20.

Anonymous (1998). Silvia Ana: Results of First Year's Service. Ship \& Boat Int., Jan, 15-16.

CEHIPAR (1998). Informe OTI-2135-CM-2 Resultados del programa de comportamiento en la mar Precal del Ferry TF-120 para investigación de control. Canal de Experiencias Hidrodinámicas del Pardo, Madrid.

CEHIPAR (2000): www.cehipar.es

Cruz, J.M de la (2000). Evaluation. Technical Report (in Spanish). CRIBAV-01-04 of Project TAP97-0607-C03-01 (ctb.dia.uned.es/cribav/).

Fossen, T.I. (1994). Guidance and Control of Ocean Vehicles, John Wiley.

Lewis, E.V. (1989). Principles of Naval Architecture, SNAME, New Jersey.

Lloyd, A.R.J.M. (1998). Seakeeping: Ship Behaviour in Rough Water, Ellis Horwood (Ed), RPM, Sussex, UK.

López, R., Santos, M. (2002). Neuro-Fuzzy modelling of a fast ferry vertical motion. Control 15 ${ }^{\text {th }}$ IFAC World Congress, Barcelona (accepted).

O’Hanlon, J.F., McCawley, M.E. (1974). Motion sickness incidence as a function of frequency and acceleration of vertical sinusoidal motion. AM.

Polo, O.R., Esteban, S., Maron, A., Grau, L., De la Cruz, J.M. (2001). Control code generation used for control experiments in ship scale model, IFAC Conference CAMS, Glasgow. 